\title{
Lingual Thyroid: A rare case report
}

\author{
S. Bhadoria, ${ }^{1}$ A. Lavania, ${ }^{2}$ R. Saxena, ${ }^{3}$ \\ ${ }^{1}$ Senior Resident, ${ }^{2}$ Asst. Professor, ${ }^{3}$ Professor \\ Dept. E.N.T and Head \& Neck Surgery, School of Medical Science \& Research, Greater Noida,Uttar Pradesh, India
}

\begin{abstract}
Lingual Thyroid is rare developmental anomaly usually affecting females it is usually located in midline, in base of the tongue and represent the faulty migration of thyroid gland ${ }^{1}$.although the exact pathogenesis of this ectopic accessory thyroid tissue is not known it generally originates from the epithelial tissue of non obliterated thyroglossal ductus $^{1,2}$ in this report a case of lingual thyroid in a 28 year old patient with difficulty in swallowing and foreign body sensation complaints for 3 years is presented ultrasound showed absent Thyroid in Neck.
\end{abstract}

Key Word: Lingual thyroid, thyroid gland.

\section{Case presentation}

A 28year old patient was admitted with the complaints of difficulty in swallowing and foreign body sensation for 3 years and progressive in nature. The medical history was insignificant.Physical examination revealed $3 \times 3 \times 2 \mathrm{~cm}$ fixed hard mass that was covered with normal mucosa in the base of the tongue (fig1). Thyroid gland was non palpable and a X Ray Neck lateral view showed us a smooth contour rounded density at the base of tongue extending up to the valleculae(Fig -2). The thyroid tissue was absent on thyroid Ultrasound Examination (Fig 3 \& 4). Doppler US showed us the Normal resistance arterial blood flow in neck .Direct Transoral Ultra sound over the lesion also showed a nodular mass containing cystic and necrotic mass. Histopathologic examination Photomicrograph (Fig.5

Correspondence: A. Lavania

E-mail: ajayisdiamond@rediffmail.com
\&6) shows minor salivary gland predominantly mucus type with presence of extracellular mucin. Along with this are seen in varying size of thyroid follicles with hyperplastic follicular cells along with colloid filled follicles. Overlying reactive stratified squamous epithelium are seen. According to the results of thyroid function test patient was Euthyroid T3 -73 .(82-179ng/ dl) T4 .77(4.5-12.5ng/dl) TSH 7.23 (0.4-4 m/u/ml). Other laboratory test were within normal limits. After thorough examination and doing routine as well as disease specific investigations we posted the patient for surgery. Mass was excised via oral approach. This is most favorable approach as neck incision can be avoided and cosmetically acceptable. Using Bipolar Cautery dissection was started from anterior end of the mass and removed totally by encircling incision. Intraoperative haemostasis was achieved using bipolar cautery. 
Journal of College of Medical Sciences-Nepal,2011,Vol-7,No-3

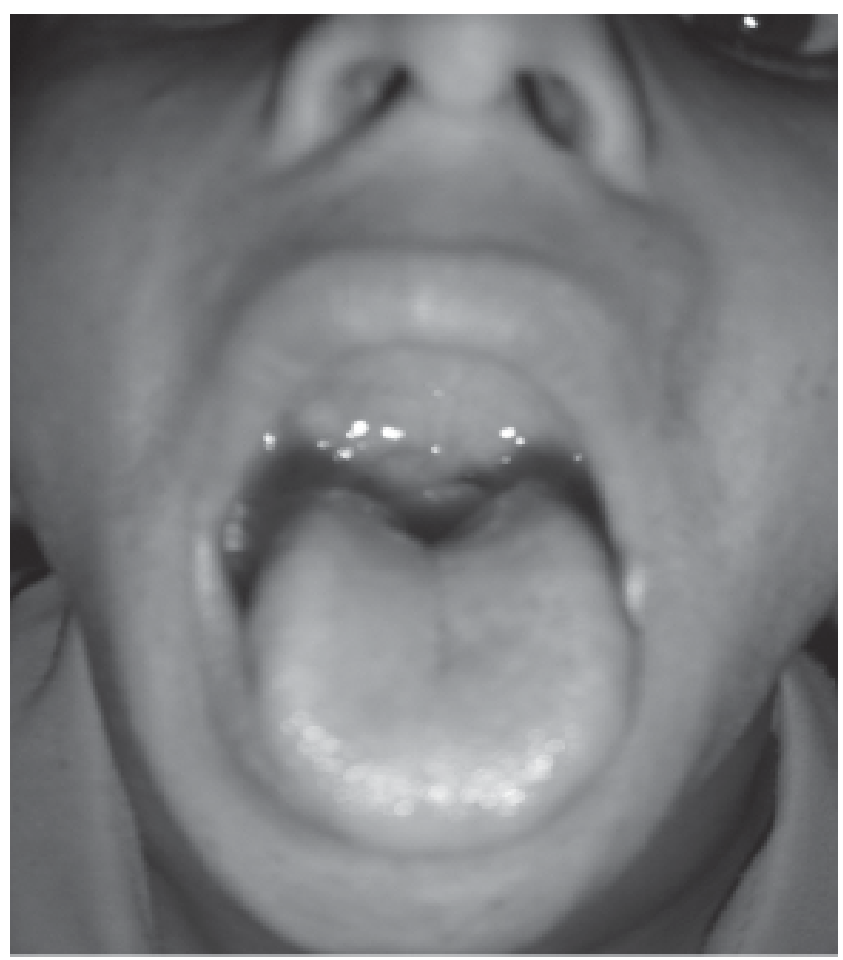

Fig. 1: Lingual Thyroid Base of Tongue

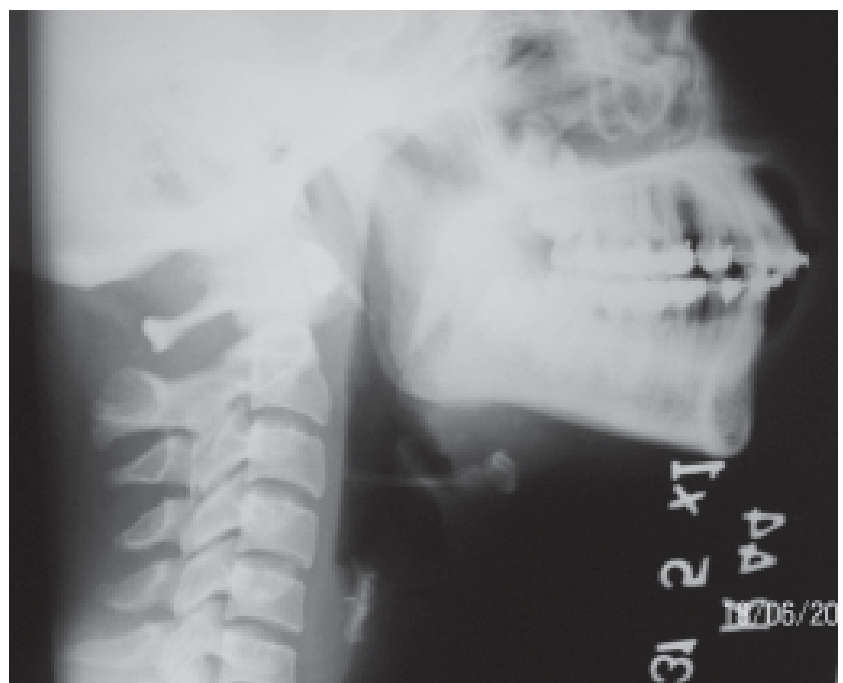

Fig. 2: XRay Neck Lateral View; Smooth Bulge at Base of Tongue

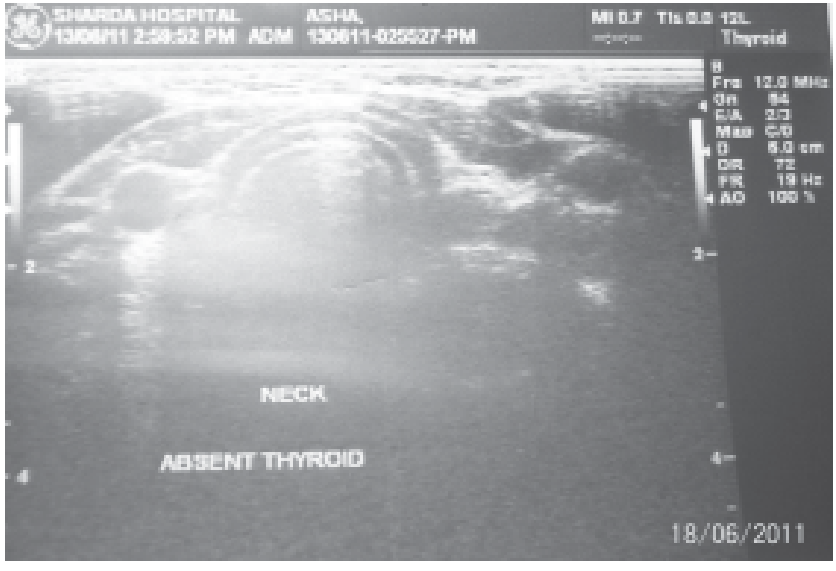

Fig 3 - USG Neck Showing Absent Thyroid

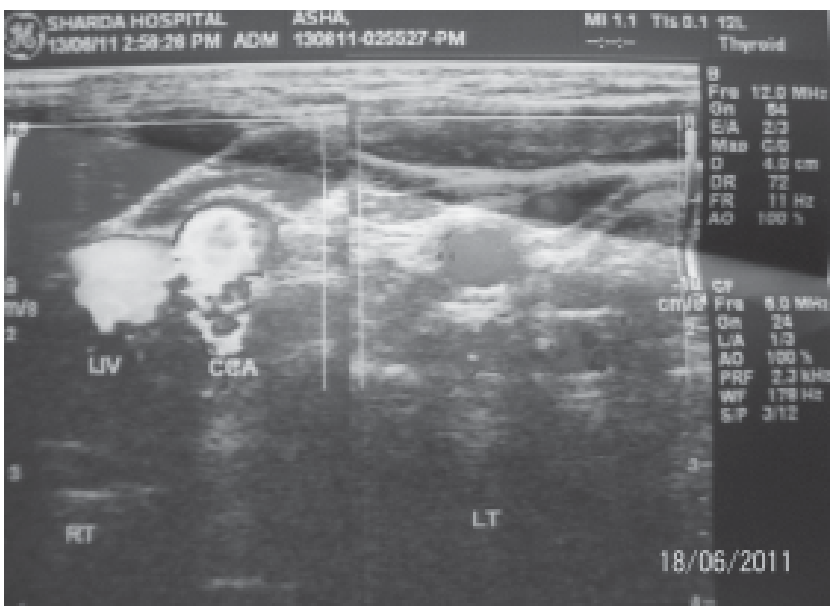

Fig 4-Doppler Neck showing Normal Blood flow in CCA

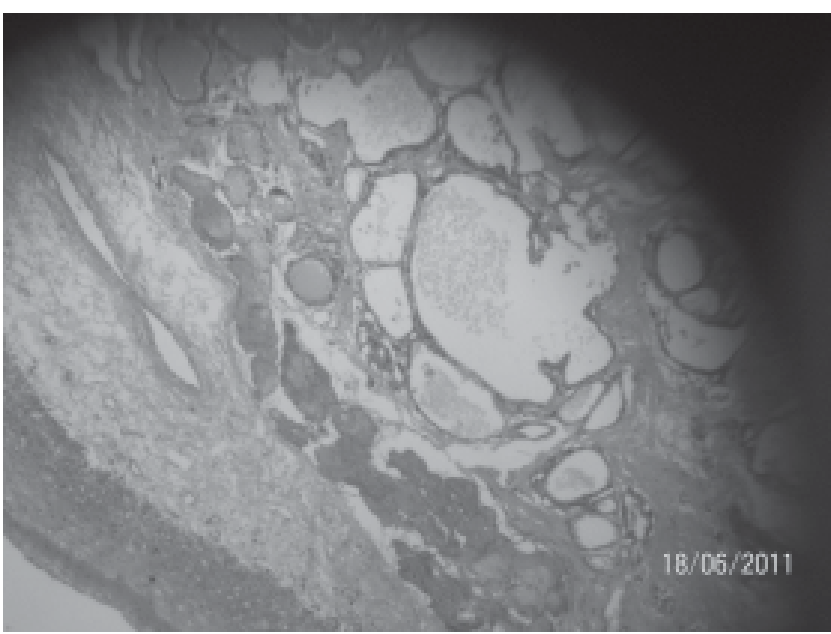

Fig 5-Thyroid Follicle with stratified Squamous Epithelium 
S. Bhadoria et al. Lingual Thyroid: A rare case report

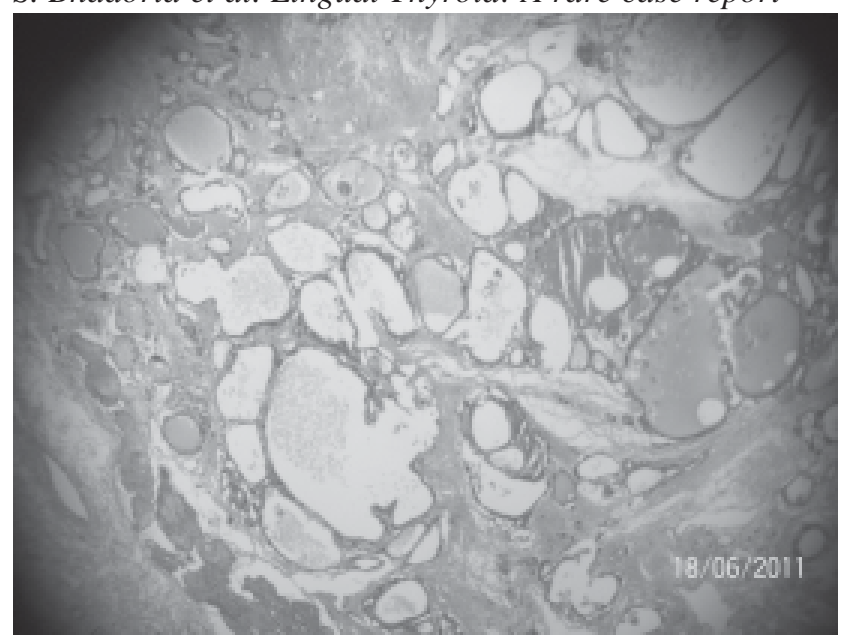

Fig 6-Thyroid Follicle with Minor Salivary

gland

Discussion

Approximately 90\% of all ectopic thyroid tissue is associated with the dorsum of the tongue. The presence of lingual thyroid reflects the lack of descent of thyroid tissue during development. The lingual thyroid is found in the midline in the area of foramen cecum. The true incidence of lingual thyroid may never be known, because this entity does not always result in clinical manifestations; however studies involving neonatal screening for hypothyroidism revealed that approximately $1 / 18,000$ to $1 / 100000$ live births were associated with ectopic thyroid tissue involving the tongue ${ }^{3,5}$.Although usually asymptomatic ,the presence of lingual thyroid can be associated with hypothyroidism .Studies have shown that upto70\% of patients with lingual thyroid also have hypothyroidism ${ }^{4}$.Other symptoms might also be related to the mass effect of the lingual Thyroid and might cause airway obstruction and/or difficulties with swallowing. Patients may complain of dysphagia or sensation of lump in the throat .Less common complaints include dysphonia or bleeding.
Symptoms may occur at times of increased metabolic demands such as growth spurts during adolescence or duringPregnancy ${ }^{7}$.Malignant transformation is rare ${ }^{8}$ Treatment of hypothyroid patients involves thyroid replacement therapy, which may also reduce the size of the lingual thyroid and, in turn, reduce any obstructive symptoms. Treatment for symptomatic euthyroid typically involves surgical excision. A number of different approaches for surgical excision of lingual thyroid have been described and include Transcervical approach by means of lateral pharyngotomy approach .or trans hyoid pharyngotomy approach as well as trans oral excision with use of the $\mathrm{CO} 2$ laser $^{6}$

\section{Conclusion:}

Ectopic lingual thyroid is an uncommon embryological aberration characterised by the presence of thyroid tissue located in a site other than the normal, pretracheal region. The tongue is the most frequent ectopic location of the thyroid gland; the clinical incidence of lingual thyroid varies between 1:3000 and 1:10,000. We present a rare case of lingual thyroid in a 28 year-old Female who presented with dysphagia. The radiological findings and the biopsy confirmed the diagnosis. Surgical excision of the mass was done using bipolar Cautery. At this time, there are no signs of complications or malignant transformation. This case is more rarer as Thyroid gland was found to be absent in the neck and confirmed by Ultrasonography.

\section{Reference}

1. P.S. Douglas, A.W. Baker . Lingual Thyroid . Br J.Oral Maxillofac. Surg. 1994;32:123-4 
Journal of College of Medical Sciences-Nepal,2011,Vol-7,No-3

2. J.G.Batsakis, A.K. EL-Naggar, M.A. Luna: Thyroid gland ectopias. Ann otol Rhinol laryngol 1996;105:9961000

3. D. Fisher, J. Dussault, T. Foley et al:Screening for Congenital Hypothyroidism: results of screening one million North American infants .J.Paediatr1979; 94:700.

4. P. Kansal. L. Sakati: Nasoalveolar cysts, J Otolaryngo1994; 123:194.

5. E.L. Kaplan:thyroid and Parathyroid. InSchwartz SI,Shires G T Spencer FC, Editors : Principles of surgery, ed5, New York1969,McGraw-Hill,1614.
6. R. Paxedder, C.L. Pelagatti, P. Nicolai: Lingual thyroid :endoscopic management with CO2 laer,Am J Otolaryngol 1998;19:136.

7. K. Taibah, M. Ahmed, E.Baessa :An unusual cause of obstructive sleep Apnoeapresenting during Pregnancy , J Laryngol Otol 1998;112:1189.

8. C.P. Winslow,E.C. Weisberger: Lingual Thyroid and neoplastic Change:a review of the literature and description of a case ,Otolaryngol head neck Surg 1997;117:8100. 